\title{
Comparison of the aphicidal activities of three chemical drugs imidacloprid, deltamethrin, and cypermethrin against the bamboo aphid Pseudoregma bambucicola (Takahashi, 1921)
}

\author{
Xiang Nong ${ }^{\mathrm{a}}$ \\ Leshan normal university \\ College of life science \\ Le'shan 614000, China \\ E-mail: nongx2008@163.com
}

\author{
Jia-Ning $\mathrm{Mao}^{\mathrm{a}}$ \\ Leshan normal university \\ College of life science \\ Le'shan 614000, China \\ E-mail:mjn2605700@163.com
}

Tian-Fei Liu

Guangdong academy of agricultural sciences

Guang'zhou 510640, China

E-mail:58771599@qq.com

\author{
Yao-Jun Yang* \\ Leshan normal university \\ College of life science \\ Le'shan 614000, China \\ E-mail: yangyaojun68@163.com; rsyyj@126.com
}

\author{
Zi Liang \\ Leshan normal university \\ College of life science \\ Le'shan 614000, China \\ E-mail: 120564533@qq.com
}

*Corresponding author

a Both authors contributed equally to this work

\begin{abstract}
Pseudoregma bambucicola is a highly reproductive bamboo aphid that affects the normal growth of bamboos, induces the formation of sooty molds, and even causes substantial injury or death among young bamboos. Objective:To identify the most effective chemical synthesis of drugs to control this aphid. Methods: This study compared the aphicidal activities of three prevention and treatment drugs, namely, cypermethrin, deltamethrin, and imidacloprid, against $\boldsymbol{P}$. bambusicola. Results: Imidacloprid demonstrated the greatest insecticidal efficacy in the in vitro experiments by killing $83.33 \%$ of the aphids within $6 \mathrm{~h}$. Cypermethrin and deltamethrin only killed $33.33 \%$ and 70\% of the aphids, respectively, within the same duration. Therefore, we used deltamethrin and imidacloprid in the field test. The amount of aphids significantly decreased four days after applying deltamethrin, and the treatment rate reached $39.67 \%$. The amount of bamboo aphids was significantly reduced in the second treatment. The treatment rate reached $81.67 \%$ on day 12 and reached $100 \%$ on day 30 . Favorable results were achieved in the imidacloprid group, in which the amount of bamboo aphids was significantly decreased, and a $100 \%$ treatment rate was achieved on the 12th day after the treatment. Conclusion: Imidacloprid demonstrates the best aphicidal activity.
\end{abstract}

Keywords- imidacloprid; deltamethrin; cypermethrin; Pseudoregma bambucicola; filed test

\section{I.INTRODUCTION}

Aphids, also known as plant lice, suck juices from plants as nutrients and are among the most important pests in many crops and plants worldwide. Aphids mainly cause damage to crops and plants, and such damage eventually results in a reduction in crop production and plant quality ${ }^{[1-3]}$. Pseudoregma bambucicola is widely found in the warm areas of eastern Asia. These aphids live and breed in bamboos to develop a large and dense population ${ }^{[4]}$

P. bambucicola seriously affects the normal growth of bamboos by sucking the sap from bamboo shoots and stems. After being infected by P. bambucicola, bamboos atrophy, turn brown, form sooty blotches that affect soil permeability, and emit a strong odor that causes air pollution. P. bambucicola can also cause the shedding of bamboo leaves and, in serious infections, lead to the death of bamboos ${ }^{[5]}$. Some people have bred special animals and insects to control the growth of aphids ${ }^{[6]}$. Ladybirds, harmonia axyridis, turtles, chrysopa syrphus flies, and aphid fungi are among the natural enemies of aphids that can be bred and used to control the growth of such pests. Although effective, the breeding of such animals and insects has indirectly caused other problems.Considering issues on safety, several researchers have investigated the use of plant source pesticides for controlling aphid growth ${ }^{[7-9]}$. Plant source pesticides, which are classified as biological pesticides, comprise effective components that are extracted from plants. Special plants, such as camellia, pepper, and ferns 
[10-11], contain secondary metabolites with favorable insecticidal activity. Given their high efficiency, potency, and environment safety, botanical pesticides have become a new type of pollution-free pesticides ${ }^{[12]}$. However, given the limitations in technology and equipment, the cost of producing botanical pesticides is very high, and such materials have become unsuitable for extensive use. When the treatment effect is considered, the use of chemical pesticides is considered the most common method for controlling aphid growth ${ }^{13}$. Although chemical pesticides are convenient to use and can quickly kill aphids, the long-term excessive use of these pesticides gives rise to several adverse consequences, such as livestock poisoning, environmental pollution, destruction of soil structure, groundwater pollution, and drinking water pollution. The organic phosphorus in these pesticides, as well as the spraying of organochlorine pesticides, indirectly pollutes the environment and poisons animals ${ }^{14-15}$.In the 20th century, aphid growth has been controlled by using pyrethroid drugs, such as deltamethrin, cypermethrin, and cyfluthrin. These drugs are efficient, have low residue, and have a low toxicity level toward both humans and animals. As a new and relatively ideal chemical pesticide, imidacloprid can efficiently kill aphids with a low toxicity level and residue ${ }^{[16]}$.

To identify the chemical drug that kills $P$. bambusicola most efficiently, we performed in vitro and field spraying to compare the aphicidal activities of cypermethrin, deltamethrin, and imidacloprid against $P$. bambusicola.

\section{MATERIALS AND METHODS}

\section{A. Material}

\section{1) Three chemical drugs:}

Three chemical drugs, namely, cypermethrin, deltamethrin, and imidacloprid, were purchased from the biological products company of the Yangtze River market in Leshan City.

\section{2) Aphids:}

The aphids were collected from bamboos that were naturally infected with $P$. bambucicola. Wingless parthenogenetic P. bambucicola were separated from their infected bamboos for the in vitro test. The field experiment was directly implemented in the bamboos that were naturally infected with P. bambucicola.

\section{B. Method}

\section{1) Aphicidal activity to P. bambucicola in vitro}

The methods of Nong et al. (2012) [17] and Chermenskaya et al. (2012) ${ }^{[18]}$ were adopted and slightly improved for this study. Distilled water was used to prepare recommended amounts of cypermethrin, deltamethrin, and imidacloprid concentrations. A $2 \mathrm{~mL}$ aliquot of each drug was placed on a Petri dish $(10 \mathrm{~cm}$ diameter) that contained filter paper chips to absorb the liquid. Ten $P$. bambucicola aphids were placed on the filter paper on each Petri dish and were incubated at $20 \pm$ $2{ }^{\circ} \mathrm{C}$ under $80 \% \pm 10 \%$ relative humidity. Three replicates were performed for each concentration. The viability of the $P$. bambucicola was checked regularly by poking them with a needle. The aphids that did not react to the needle were recorded as dead. The distilled water was used as an untreated control.

\section{2) Field trial}

The field experiment was performed in a bamboo grove that was severely infested with P. bambucicola. The recommended amounts of two drugs, deltamethrin and imidacloprid were used for the field experiment, and distilled water was used in the untreated control group. Each treatment was repeated thrice. Before the field experiment, we carefully selected nine bamboo trees that were severely infected with P. bambucicola. The aphid infection density was not significantly different among the plants in the location. The selected bamboo plants were randomly divided into three groups (groups A, B, and $\mathrm{C}$, respectively), with each group including three bamboo plants. Group A was the deltamethrin-treated group, group B was the imidacloprid-treated group, and group $\mathrm{C}$ was the untreated control (using distilled water) group. The selected bamboo plants were treated twice on days 0 and 4 . We used a small sprayer (especially used for TLC) for the application of deltamethrin, imidacloprid, and distilled water. After spraying, the drugs were fairly distributed on the plant, and the aphid-gathering areas were covered with the chemicals. The investigations were conducted on day 0 (before spraying) and on days 4, 8, 12, and 30 (after spraying) using a three-point and one-point sampling strategies. We chose the upper, middle, and lower parts of each aphid-gathering area (three-point sampling strategy) on days 0 and 4 , and the one-point sampling strategy was conducted on days 8, 12, and 30 . Under the sampling strategy, the aphids were collected from a $1 \mathrm{~cm} 2$ area/per point on the plant to calculate the mean aphid number. The aphid reduction rate and field treatment effect were calculated using the following formulas [19]:

Aphid Reduction Rate (IRR) $(\%)=($ Aphid number before spraying - Aphid number after spraying)/Aphid number before spraying $\times 100 \%(1)$ and

Field treatment effect $(\%)=($ IRR of treated group - IRR of control group $) /(100-$ IRR of control group $) \times 100 \%$ (2).

\section{3) Statistical analysis}

Analysis of variance tests were performed using the SAS software 20. The significance of the differences in insect mortality rates under different ageraphorone concentrations was calculated using the probability method. The median lethal time (LT50) was calculated using a complementary log-log (CLL) model. We also assessed the statistical significance of the field experiment results. 


\section{RESULTS AND ANALYSIS}

A. Aphicidal activity of the three drugs against $P$. bambucicola in vitro

A $2 \mathrm{~mL}$ aliquot of each drug was placed on a Petri dish (10 $\mathrm{cm}$ diameter) that contained filter paper chips to absorb the liquid. Ten P. bambucicola aphids were then placed on the filter paper on each Petri dish. Three replicates were performed for each concentration. Deltamethrin and imidacloprid were shown to be highly toxic to P. bambucicola (Table 1).

TABLE I. THREE DRUGS USED AGAINST P. BAMBUCICOLA IN VITRO.

\begin{tabular}{lcccc}
\hline \multicolumn{5}{c}{ Time $(\mathrm{min}) / \mathrm{Mean}$ mortality $(\%) \pm \mathrm{SE}$} \\
\cline { 2 - 5 } Drugs & $120 \mathrm{~min}$ & $180 \mathrm{~min}$ & $300 \mathrm{~min}$ & $360 \mathrm{~min}$ \\
\hline Cypermethrin & $20.00 \pm 5.77 \mathrm{~A}(\mathrm{~B})$ & $23.33 \pm 6.67 \mathrm{~A}(\mathrm{~B})$ & $30.00 \pm 5.77 \mathrm{~A}(\mathrm{~B})$ & $33.33 \pm 3.33 \mathrm{~A}(\mathrm{~B})$ \\
Deltamethrin & $20.00 \pm 5.77 \mathrm{C}(\mathrm{B})$ & $33.33 \pm 28.8 \mathrm{BC}(\mathrm{AB})$ & $63.33 \pm 12.02 \mathrm{AB}(\mathrm{A})$ & $70.00 \pm 10.00 \mathrm{~A}(\mathrm{~A})$ \\
imidacloprid & $40.00 \pm 5.77 \mathrm{~B}(\mathrm{~A})$ & $46.67 \pm 3.33 \mathrm{~B}(\mathrm{~A})$ & $56.67 \pm 6.37 \mathrm{~B}(\mathrm{~A})$ & $83.33 \pm 3.33 \mathrm{~A}(\mathrm{~A})$ \\
Untreat control & $0.00 \pm 0.00 \mathrm{~A}(\mathrm{C})$ & $3.33 \pm 3.33 \mathrm{~A}(\mathrm{C})$ & $3.33 \pm 3.33 \mathrm{~A}(\mathrm{C})$ & $3.33 \pm 3.33 \mathrm{~A}(\mathrm{C})$ \\
\hline Note: Different letters within a row denote significant differences between different times (P< 0.05$).$ Different letters in brackets within a column \\
denote significant differences between different concentrations $(\mathrm{P}<0.05)$.
\end{tabular}

Imidacloprid exhibited a satisfactory aphicidal effect on P. bambucicola. This drug achieved a $83.33 \%$ aphid mortality within $6 \mathrm{~h}$, whereas cypermethrin and deltamethrin achieved aphid mortalities of $33.33 \%$ and $70 \%$, respectively, within $6 \mathrm{~h}$ (Table 1).

\section{B. Probit regression analysis of toxicity (median lethal} time, LT50) of the three drugs in vitro

The data were computed using SAS software (SAS Institute 2002). LT50 was calculated using the CLL model. The toxicity of the three drugs against aphids in vitro was evaluated using the CLL model. Table 2 shows the LT50 values and regression lines for the three drugs against $\mathrm{P}$. Bambucicola.

TABLE II. PROBIT REGRESSION ANALYSIS OF THE TOXICITY (LT50) OF THE THREE DRUGS AGAINST APHIDS IN VITRO.

\begin{tabular}{cccc}
\hline Drugs & Regression Line & $\mathrm{LT}_{50} /(\mathrm{h})(95 \% \mathrm{FL})$ & Pearson Chi-Square \\
\hline Cypermethrin & $\mathrm{Y}=0.864 \mathrm{X}-1.119$ & $19.680(-------)$ & 4.000 \\
Deltamethrin & $\mathrm{Y}=2.979 \mathrm{X}-1.783$ & $3.968(3.269-4.938)$ & 10.035 \\
imidacloprid & $\mathrm{Y}=2.047 \mathrm{X}-0.974$ & $2.991(1.849-3.899)$ & 6.250 \\
\hline
\end{tabular}

The LT50 values of cypermethrin, deltamethrin, and imidacloprid were $19.68 \mathrm{~h}, 3.968 \mathrm{~h}$ and $2.991 \mathrm{~h}$, respectively (Table 2). The in vitro aphicidal activity of deltamethrin and imidacloprid demonstrated a time dependence.

\section{Field trial}

We used deltamethrin and imidacloprid for the field trial. Deltamethrin was used in group A, imidacloprid was used in group B, and distilled water was used in group C. The treatments were repeated thrice on each group. The number of aphids among the bamboo plants in groups A, $\mathrm{B}$, and $\mathrm{C}$ did not significantly differ prior the field experiment. In other words, the aphid infestation density was not significantly different among the three groups. The bamboo plants in group A exhibited a clear and rapid recovery after they were sprayed with deltamethrin and imidacloprid. The aphid density area was significantly reduced on days 4 and 8 (Fig .1; A2, and A3). Nearly all of the infested plants in group A $(99.33 \%)$ recovered on day 12. No recurring infestations were observed on day 30 (Table 3). The infested bamboos in the imidacloprid group (group B) exhibited an excellent improvement during the experimental period and an enhanced recovery after the second treatment (Fig .1; B2 and B3). All of the infested plants in group B $(100 \%)$ had completely recovered on days 12 to 30 . Imidacloprid demonstrated better effects against cotton aphids (Aphis gossypii) as compared with $5 \mathrm{mg} / \mathrm{mL}$ of $\mathrm{n}$-Hexane and dichloromethane extracts from C. ficifolium11. The effect of 9-Oxo-10,11-dehydro-ageraphorone against A. gossypii was similar to that of alkaloids from the seeds of $\mathrm{M}$. cordata on day 7 [20]. 
However, the bamboos in the group $\mathrm{C}$ showed no signs of recovery (Fig .1; C2 and C3). The number of aphids in this group increased on day 8 . The bamboos in groups $\mathrm{A}$ and $\mathrm{B}$ did not show any presence of aphids on day 30. Therefore, deltamethrin and imidacloprid achieved a $100 \%$ field efficacy rate according to the abovementioned formula.

TABLE III. TREATMENT EFFECT IN THE THREE GROUPS (MEAN \pm SE).

\begin{tabular}{lccccc}
\hline Groups & 0 day & 4 day & \multicolumn{2}{c}{8 day } & \multicolumn{2}{c}{12 day } & \multicolumn{2}{c}{30 day } \\
\hline A: deltamethrin & $0.00 \pm 0.00 \mathrm{D}(\mathrm{A})$ & $39.67 \pm 0.88 \mathrm{C}(\mathrm{A})$ & $81.67 \pm 2.03 \mathrm{~B}(\mathrm{~A})$ & $99.33 \pm 0.67 \mathrm{~A}(\mathrm{~A})$ & $100.00 \pm 0.00 \mathrm{~A}(\mathrm{~A})$ \\
B: imidacloprid & $0.00 \pm 0.00 \mathrm{D}(\mathrm{A})$ & $51.00 \pm 2.31 \mathrm{C}(\mathrm{A})$ & $90.00 \pm 5.29 \mathrm{~B}(\mathrm{~A})$ & $100.00 \pm 0.00 \mathrm{~A}(\mathrm{~A})$ & $100.00 \pm 0.00 \mathrm{~A}(\mathrm{~A})$ \\
C: Untreated & $0.00 \pm 0.00 \mathrm{~A}(\mathrm{~A})$ & $17.00 \pm 9.54 \mathrm{~A}(\mathrm{~B})$ & $12.33 \pm 7.88 \mathrm{~A}(\mathrm{~B})$ & $7.17 \pm 6.43 \mathrm{~A}(\mathrm{~B})$ & $6.00 \pm 6.00 \mathrm{~A}(\mathrm{~B})$ \\
\hline
\end{tabular}

*Note: The different letters within a row denote the significant variances between different periods $(\mathrm{P}<0.05)$. The different letters that were enclosed in brackets within a column denote the significant variances between different concentrations $(\mathrm{P}<0.05)$

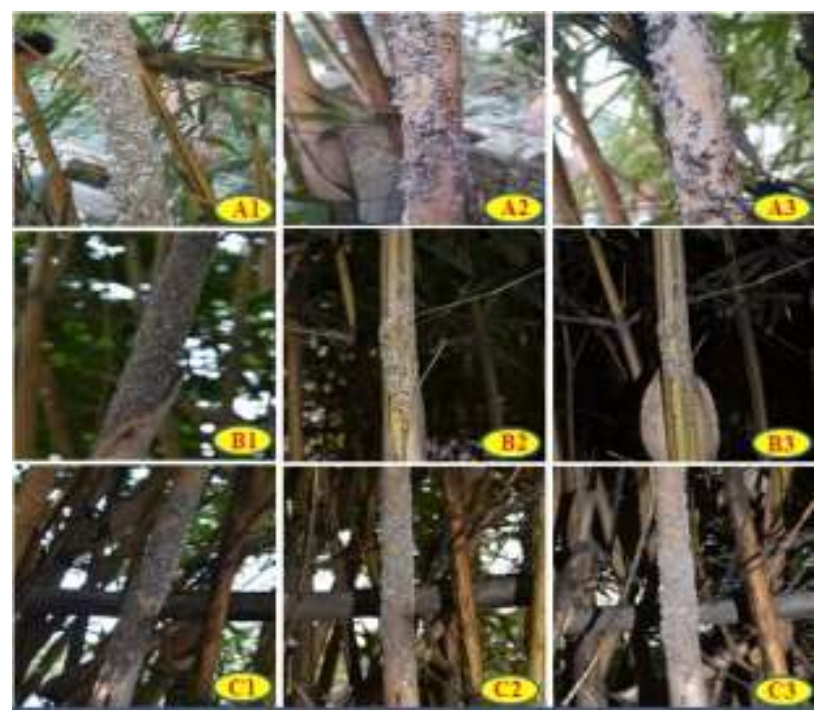

Figure 1. Treatment effects on the infected bamboos.

*Note: Group A denotes the infected regions that are treated with deltamethrin. Groups B (treated with imidacloprid) and C (untreated control) show the infected regions. A1, B1, and C1 show the signs of aphid infection before treatment. A2, B2, and C2 show the observations on plant recovery on day 4 . $\mathrm{A} 3, \mathrm{~B} 3$, and $\mathrm{C} 3$ show the observations on plant recovery on day 8 .

\section{DISCUSSION}

Aphid growth is currently being controlled by using chemical pesticides. Although many low-toxicity pesticides can be used for controlling aphid growth, the number of effective pesticides is very limited [21]. Some pesticides are not stable enough, produce residues, or cause harm to the body. Pesticides with CIS cypermethrin must not be used as they are harmful to humans.Deltamethrin, cyermethrin, and imidacloprid demonstrated favorable effects against P. bambucicola. These pesticides have low toxicity, low residues, and favorable insecticidal effects. Imidacloprid demonstrated the best aphicidal activity. Imidacloprid (1-(6 chloro-3-pyridyl methyl)-N-nitro imidazole-2- subunit amine) is a new type of systemic insecticide that can effectively control aphids, whiteflies, psyllids, bugs, and other harmful pests [22]. Imidacloprid is highly effective against Myzus persicae and can prevent the formation of wheat aphids.After treatment with imidacloprid, the aphids demonstrated intensified activities, quickly climbed the bamboo rods, suffered from severe convulsions, entered paralysis, and fell to their death. Given its low toxicity, lack of odor, and low dosage, imidacloprid does not pose any harm to humans and livestock, as well as protects the natural enemies of P. bambusicola, such as ladybirds and Syrphidae 1. Imidacloprid is the ideal prevention and treatment measure for $\mathrm{P}$. bambucicola because of its durability and security.

\section{ACKNOWLEDGMENTS}

This study was supported by grants from the Leshan Normal University (No. Z1327), and Science \& Technology Department of Sichuan Province (No. 2014NZ0012, 2015JY0133).

\section{REFERENCES}

[1] Activit Kim, S. K., Kim, Y.C., Lee, S., Kim, J.C., Yun, M. Y., Kim, I. S. J. Agric. Food Chem. 59,934-938 (2011).

[2] Baek, M. Y., Park, H. J., Kim, G. M., Lee, D. Y., Lee, G. Y., Moon, S. J., Ahn, E. M., Kim, G. S., Bang, M. H., Baek, N. I. J Korean Soc Appl Biol Chem .2013.56, pp:135-140.

[3] Rod, S. A. Southwest Form Press. 2014.41(6),pp: 19.

[4] Fukatsu, T., Shibao, H., Nikoh, N., Aoki, S. Molecular Phylogenetics and Evolution.2001.18(3), pp. 423-433. 
[5] Petitt, F. and Smilowitz, Z. Journal of Economic Entomology, 75, 431-435.(1982).

[6] Deng Shun, Wang Peng, Shu Jin-Ping, Wang Hao-Jie. Journal of environmental entomology. 2009.31(4),pp: 300-305.

[7] Pavela, R., Journal of Essential Oil Bearing Plants, 2006, 9:2, pp: 99-106.

[8] Dang, Q. L., Lee, G. Y., Choi, Y. H., Choi, G. J., Jang, K. S., Park, M. S., Soh, H. S., Han, Y. H., Lim, C. H., Kim, J. C. Crop Protection 2010. 29, pp:1124-1129.

[9] Rashid, T., Chen, J., McLeod, P., Toxicity. 2013.1(2), pp: 20-23.

[10] Habou, Z. A., Haougui, A., Mergeai, G., Haubruge, E., Toudou, A., Verheggen, F. J. TROPICULTURA, 2011.29(4), pp: 225-229.

[11] Park, H. J., Baek, M. Y., Cho, J. G., Seo, K. H., Lee, G. Y., Moon, S. J., Baek, N. I., J. Korean Soc. Appl. Biol. Chem. 2011, 54(3),pp: 345-352.

[12] Panella, N. A., Dolan,M. C., Karchesy,J. J., Xiong,Y. P., Peralta-cruz,J., Khasawneh,M., Montenieri,J.A Maupin,G.O. J.Med. Entomol. 2005, 42, pp: 352-358.

[13] Mccarville, M. T., O’Neal, M. E. Journal of Economic Entomology, 2013,106(3), pp:1302-1309.

[14] Dong, S. L, Andrews, M, C, Moores, G, D, Han, Z. J, Williamson, M, S. Chemico-Biological Interactions. 2005, 157,pp: 373-374.
[15] Ma, Guo-Lan, Bai Lian-Yang. Journal of Anhui Agriculture Sciences. 2006, 34(13), pp: 3406-3408.

[16] Yi Long. Journal of the botany doctor.2003, 16 (3), pp: 25-26. (In Chinese).

[17] Nong, X., Fang, C. L., Wang, J. H., Gu, X. B., Yang, D. Y., Yang, G. Y., et al. Veterinary Parasitology. 2012.187, pp: 345-349.

[18] Chermenskaya, T. D., Stepanycheva, E. A., Shchenikova, A. V., Savelieva, E. I., Chakaeva, A. S.. Industrial Crops and Products 2012, 36 ,pp: 122-126.

[19] Xu,R., Wu,D., Zhang,W.D., Yin,F., Kuang,R.P. International Journal of Pest Management.2009, 55, (4),pp: 151-156.

[20] SAS Institute. SAS Procedures Guide. Version 9. SAS Institute, Cary, NC.2002

[21] Kim, J.J, Seo, D. K, Kim, G. H. Korean journal of applied entomology. 2006, 45(2), pp: 217-226.

[22] Kumar J , N. A. Shakil, M. A. Khan, Kamlesh Malik \& Suresh Walia. Journal of Environmental Science and Health, Part B:Pesticides, Food Contaminants, and Agricultural Wastes, 2011, 46:8, pp: 678-682. 